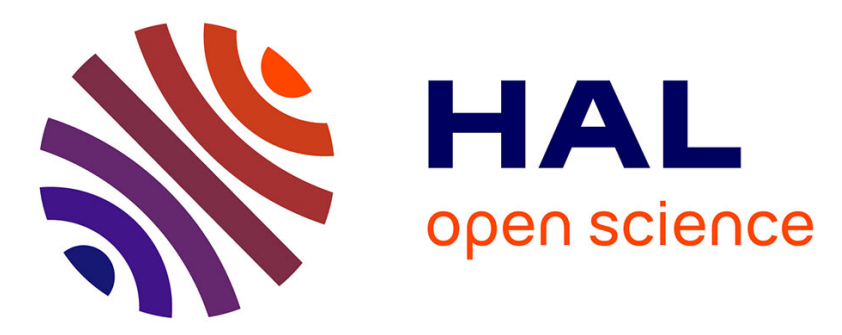

\title{
Determining Nonlinear Optical Coefficients of Metals by Multiple Angle of Incidence Heterodyne-Detected Sum-Frequency Generation Spectroscopy
}

\author{
Wei-Chen Yang, Bertrand Busson, Dennis K Hore
}

\section{- To cite this version:}

Wei-Chen Yang, Bertrand Busson, Dennis K Hore. Determining Nonlinear Optical Coefficients of Metals by Multiple Angle of Incidence Heterodyne-Detected Sum-Frequency Generation Spectroscopy. Journal of Chemical Physics, 2020, 152, pp.084708. 10.1063/1.5133673 . hal-02471034

\author{
HAL Id: hal-02471034 \\ https://hal.science/hal-02471034
}

Submitted on 7 Feb 2020

HAL is a multi-disciplinary open access archive for the deposit and dissemination of scientific research documents, whether they are published or not. The documents may come from teaching and research institutions in France or abroad, or from public or private research centers.
L'archive ouverte pluridisciplinaire HAL, est destinée au dépôt et à la diffusion de documents scientifiques de niveau recherche, publiés ou non, émanant des établissements d'enseignement et de recherche français ou étrangers, des laboratoires publics ou privés. 


\section{Determining Nonlinear Optical Coefficients of Metals by Multiple Angle of Incidence Heterodyne-Detected Sum-Frequency Generation Spectroscopy}

Wei-Chen Yang, ${ }^{1}$ Bertrand Busson, ${ }^{2}$ and Dennis K. Hore ${ }^{1,3}$

1) Department of Chemistry, University of Victoria, Victoria, British Columbia, V8W 3V6, Canada

2) Université Paris-Saclay, CNRS, Institut de Chimie Physique, UMR 8000, 91405 ORSAY, France ${ }^{\mathrm{a})}$

3) Department of Computer Science, University of Victoria, Victoria, British Columbia, V8W 3P6, Canada ${ }^{\text {b) }}$

(Dated: 7 February 2020)

We illustrate a technique by which heterodyne-detected sum-frequency generation spectroscopy is performed at multiple angles of incidence in order to decompose components of the second-order susceptibility tensor when all beams are polarized parallel to the plane of incidence. As an illustration we study the non-vibrationally resonant gold response. We benchmark our results by comparing with measurements obtained in a polarization scheme that isolates a single element of the susceptibility tensor. Our technique is particularly valuable in the case of metal substrates, where the surface selection rule often prevents spectra from being acquired in multiple beam polarizations.

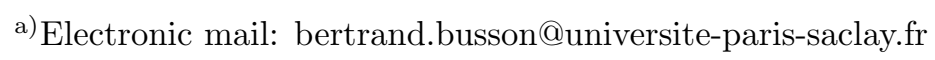

b) Electronic mail: dkhore@uvic.ca 


\section{INTRODUCTION}

Visible-infrared sum-frequency generation is a second-order nonlinear vibrational spectroscopy technique that is valued for its ability to probe the structure of molecules at solid, liquid, and vapor interfaces. ${ }^{1-3}$ Under the electric dipole approximation, the second-order electric susceptibility $\chi^{(2)}$ is non-zero only in the absence of inversion symmetry. ${ }^{4,5}$ Therefore, when a broadband or tunable infrared beam is spatially- and temporally-overlapped with a fixed frequency visible or near-infrared beam, vibrational resonances in $\chi^{(2)}$ of surface species may be observed. Performing the experiment with different beam polarizations enables different elements of the rank-3 $\chi^{(2)}$ tensor to be obtained. If the orientation of a chemical functional group is of interest, measurements are typically carried out in at least two polarization schemes. For example, an experiment in which the s-polarized component of the SFG is measured when s-polarized visible and p-polarized infrared is used (the socalled ssp scheme) selectively probes $\chi_{y y z}^{(2)}$ (see Fig. 1). Similarly, sps experiments provide access to $\chi_{y z y}^{(2)}$. If we consider the methyl symmetric stretch, the orientation of its $C_{3}$ axis may be determined from the ratio $\chi_{y y z}^{(2)} / \chi_{y z y}^{(2)}$. There are many descriptions in the literature that provide details on this procedure. ${ }^{6-12}$

Collecting spectra in multiple polarization schemes generally works well for dielectric interfaces, keeping in mind that the surface fields are enhanced or reduced at specific angles of incidence, and the optimum settings depend on the polarization of all beams. ${ }^{13-15}$ In the case of metals, however, the amplitude of s-polarized light (electric field vector perpendicular to the plane of incidence) at the surface is strongly reduced. The extent to which the surface field decreases is dependent on the metal and specific frequency, but often creates the situation where only the ppp polarization scheme yields appreciable signals. This presents a challenge for orientation analysis as $\chi_{x x z}^{(2)}, \chi_{x z x}^{(2)}, \chi_{z x x}^{(2)}$ and $\chi_{z z z}^{(2)}$ are all potential contributors to the measured response. If the nature of the surface species and the vibrational assignment are well understood, then one option is to perform homodyne (intensity) measurements of ppp spectra at multiple angles of incidence and then simultaneously solve for the amplitudes of interest by assuming a specific lineshape, such as a Lorentzian. ${ }^{16-19}$ For samples that generate response with both s- and p-components of the incident fields, there are methods that can extract the $\chi^{(2)}$ tensor elements by varying the beam polarizations. ${ }^{20,21}$

Here we present an approach that is universally applicable, can measure complex-valued 
$\chi^{(2)}$ elements, and is able to provide the dispersion of these quantities throughout the region over which the laser frequencies are tuned. We demonstrate our method applied to the case of the gold surface, of interest to many surface studies. ${ }^{22-26}$ The choice of gold is further motivated by its universal use as a support for (e.g. thiolated) self-assembled molecular layers, its chemical stability in air and its intense SFG nonresonant response (due to contributions

from its free and bound electrons. ${ }^{27,28}$ ) We begin by introducing some formalism that relates the surface fields to the incidence and reflected SFG fields. We also describe the quadrupolar response from the bulk of the material. We then analyze heterodyne-detected ssp SFG data to obtain the magnitude and phase of $\chi_{y y z}^{(2)}$ from a gold surface when the visible laser is fixed at $532 \mathrm{~nm}$, and the infrared beam has a frequency of $2800 \mathrm{~cm}^{-1}$. We use these results to verify the outcome of our analysis of multi-angle heterodyne ppp experiment on gold from 40-70 . In the end, our method is applicable to any dielectric or metal surface, but is particularly valuable when structural information is sought and two or three independent $\chi^{(2)}$ elements must be extracted from ppp data.

\section{BACKGROUND}

\section{A. Nonlinear optical response of metal surface and bulk in SFG}

Before turning to the description of our heterodyne angle-dependent method, we first review the essential nonlinear properties of gold in order to determine which tensor components have to be taken into account in the data analysis. The second-order nonlinear optical response of a metal surface has been studied soon after the first reported evidence of second harmonic generation $(\mathrm{SHG}),{ }^{29-31}$ and continues to be of interest in the SHG and SFG community. ${ }^{32-40}$ The description of the nonlinear gold response presented here follows from the general phenomenological model of surface and bulk SFG response, ${ }^{27,29,41-43}$ analogous to SHG models, such as that developed by Mizrahi and Sipe. ${ }^{44}$ For a polycrystalline cubic material, the bulk and surface behave essentially like an isotropic system. The surface second-order polarization has its origins in the discontinuity of the electron density while crossing the interface at $z=0$ (see Fig. 1), creating large electric field gradients. ${ }^{42,43}$ The resulting surface nonlinear polarization is obtained by integration of the bulk polarization along $z$ across the interface. ${ }^{41}$ The surface terms of the gold second-order susceptibility 


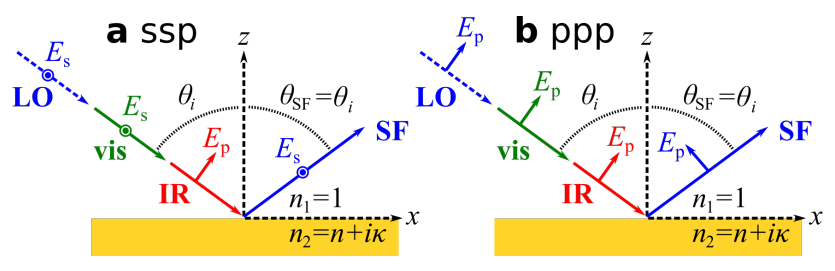

FIG. 1. Definition of the coordinate system and conventions used in this analysis for the (a) ssp and (b) ppp polarization schemes. $z$ is along the surface normal, $(x, y)$ define the interface between air and gold, and $(x, z)$ is the plane of incidence.

$\chi_{i j k, \text { surf }}^{(2)}$, defined as $P_{\mathrm{i}}^{S}=\chi_{i j k, \text { surf }}^{(2)} E_{\mathrm{vis}, \mathrm{j}} E_{\mathrm{IR}, \mathrm{k}}$ have $C_{\infty v}$ symmetry, leaving only the usual seven non-vanishing terms $z z z, x x z=y y z, x z x=y z y$ and $z x x=z y y$ (Table I). ${ }^{45}$ The parallel components of the electric fields experience no gradients as they are continuous across the interface, so the surface $z x x$ term vanishes. ${ }^{43,46}$

Apart from these surface-specific contributions, the emitted SFG also contains contributions from bulk effects, which we present in more detail as their properties fundamentally differ from the well-known surface terms. In isotropic materials, they arise from the gradients associated with the propagation of the light waves (electric fields $\mathbf{E}_{\mathrm{vis}}$ and $\mathbf{E}_{\mathrm{IR}}$ ) inside the bulk, and therefore depend on their bulk wavevectors $\mathbf{q}_{\mathrm{vis}}$ and $\mathbf{q}_{\mathrm{IR}}$ where $\mathbf{q}_{i}=q_{i, x} \hat{x}-q_{i, z} \hat{z}$ and

$$
\begin{aligned}
q_{i, x} & =\frac{\omega_{i}}{c} \sin \theta_{i}=\frac{n_{i} \omega_{i}}{c} \sin \theta_{i}^{T}, \\
q_{i, z} & =n_{i} \frac{\omega_{i}}{c} \cos \theta_{i}^{T},
\end{aligned}
$$

where $n_{i}$ is the bulk refractive index at $\omega_{i}$ (refractive index of air is taken as unity), and $\theta_{i}$ and $\theta_{i}^{T}$ are the angles of incidence of beam $i$ in air and in the bulk, respectively. The situation is different when bulk dipolar contributions are allowed, for example in a chiral liquid, ${ }^{47,48}$ as they will dominate the quadrupolar response due to the gradients. The penetration of light inside the gold is limited by the large value of the attenuation coefficient: at $532 \mathrm{~nm}$, the electric field decays to $1 / \mathrm{e}$ of its initial value after $37 \mathrm{~nm}$; at $2800 \mathrm{~cm}^{-1}$ the corresponding value is $23 \mathrm{~nm}$. However, considering fcc gold with a lattice constant around $0.4 \mathrm{~nm}$, the penetration depth is greater than 50 unit cells. The bulk terms are therefore important to consider. In addition, large attenuation results in large wavevectors in Eq. 1, leading to large field gradients as will be discussed later (Eq. 3).

In the literature, the comparison between surface and bulk terms has been extensively 
studied for SHG, ${ }^{49,50}$ but less so for SFG. ${ }^{4,51-57}$ There are two ways to describe the bulk contributions from an isotropic medium. The first one is inspired from the original work of Bloembergen and Pershan, ${ }^{29}$ separating the nonlinear polarization inside the bulk into components parallel and perpendicular to the source wavevector $\mathbf{q}_{\mathrm{vis}}+\mathbf{q}_{\mathrm{IR}}$. Each component radiates in the reflected and transmitted directions as deduced from the boundary conditions at the interface. This formulation leads to a single, compact but intricate, bulk contribution to the effective nonlinear susceptibility that is added to the surface terms. ${ }^{27,42,43}$ In the isotropic case, the bulk source is parametrized by the non-vanishing coefficients of the quadrupolar nonlinear susceptibility defined by

$$
\mathbf{P}^{\mathrm{B}}\left(\mathbf{q}_{\mathrm{vis}}, \mathbf{q}_{\mathrm{IR}}, \mathbf{r}\right)=\mathbf{P}^{\mathrm{B}}\left(\mathbf{q}_{\mathrm{vis}}, \mathbf{q}_{\mathrm{IR}}\right) \exp \left[i\left(\mathbf{q}_{\mathrm{vis}}+\mathbf{q}_{\mathrm{IR}}\right) \cdot \mathbf{r}\right]
$$

with

$$
\begin{aligned}
\mathbf{P}^{\mathbf{B}}\left(\mathbf{q}_{\mathrm{vis}}, \mathbf{q}_{\mathrm{IR}}\right)= & i\left[D_{\mathrm{vis}} \mathbf{q}_{\mathrm{vis}}\left(\mathbf{E}_{\mathrm{vis}} \cdot \mathbf{E}_{\mathrm{IR}}\right)+\Delta_{\mathrm{vis}}\left(\mathbf{q}_{\mathrm{vis}} \cdot \mathbf{E}_{\mathrm{IR}}\right) \mathbf{E}_{\mathrm{vis}}\right. \\
& \left.+D_{\mathrm{IR}} \mathbf{q}_{\mathrm{IR}}\left(\mathbf{E}_{\mathrm{vis}} \cdot \mathbf{E}_{\mathrm{IR}}\right)+\Delta_{\mathrm{IR}}\left(\mathbf{q}_{\mathrm{IR}} \cdot \mathbf{E}_{\mathrm{vis}}\right) \mathbf{E}_{\mathrm{IR}}\right]
\end{aligned}
$$

under the plane wave hypothesis $\mathbf{q}_{i} \cdot \mathbf{E}_{i}=0$. Analogous coefficients have long been defined in $\mathrm{SHG}^{58,59}$ and extension to crystalline cubic materials is possible by adding anisotropic terms. ${ }^{60,61}$ A quantitative evaluation of these coefficients is necessary to determine the impact of the bulk response as compared to the surface terms. For this purpose, simple models enable calculation of the $D_{i}$ and $\Delta_{i}$ coefficients in Eq. 3 from the electronic properties of gold, ${ }^{43}$ while a more rigorous description requires separating the contributions from free and bound electrons. ${ }^{27,62}$ Surface and bulk effective susceptibilities, calculated separately, reconstruct the total SFG response from gold. ${ }^{28}$

The second formulation for the bulk terms describes the bulk as an infinite stack of thin plates, just like the surface terms. Considering that the bulk polarization in Eq. 2 is independent of the position except for its phase term, each plate radiates the same electric field as if it were located at $z=0$, taking into account the depth-dependent phase shift $\Delta \phi=(\Delta \mathbf{q})_{\mathrm{R}} \cdot \mathbf{r}$ where $(\Delta \mathbf{q})_{\mathrm{R}}=\mathbf{q}_{\mathrm{vis}}+\mathbf{q}_{\mathrm{IR}}-\mathbf{q}_{\mathrm{SFG}}$, with $\mathbf{q}_{\mathrm{SFG}}=q_{\mathrm{SFG}, x} \hat{x}+q_{\mathrm{SFG}, z} \hat{z}$ in reflection. Because of momentum conservation parallel to the surface, ${ }^{29}$ the wavevector mismatch $\Delta \mathbf{q}_{\mathrm{R}}$ applies to only the $z$-components of the wavevectors and $\Delta \mathbf{q}_{R}=-\left(\Delta q_{z}\right)_{\mathrm{R}} \hat{z}$. Integration along $z$ produces an equivalent surface contribution defined by ${ }^{4,53}$

$$
\mathbf{P}^{\mathrm{B}, \text { surf }}\left(\mathbf{q}_{\mathrm{vis}}, \mathbf{q}_{\mathrm{IR}}\right)=\frac{i \mathbf{P}^{\mathrm{B}}\left(\mathbf{q}_{\mathrm{vis}}, \mathbf{q}_{\mathrm{IR}}\right)}{\left(\Delta q_{z}\right)_{\mathrm{R}}}
$$




\begin{tabular}{|c|c|c|c|c|c|}
\hline $\begin{array}{l}\text { tensor } \\
\text { element }\end{array}$ & polarization & $\begin{array}{c}\text { dipolar } \\
\text { surface term }\end{array}$ & $\begin{array}{c}\text { inseparable } \\
\text { term }\end{array}$ & $\begin{array}{c}\text { separable bulk } \\
\text { (non-separable as } \\
\text { a function of angle) }\end{array}$ & $\begin{array}{c}\text { separable bulk } \\
\text { (separable as } \\
\text { a function of angle) }\end{array}$ \\
\hline$x x x$ & ppp & - & - & - & yes (1.1) \\
\hline$x z z$ & ppp & - & - & - & yes $(0.008)$ \\
\hline$z z x$ & ppp & - & - & - & yes $(0.008)$ \\
\hline$z x z$ & ppp & - & - & - & yes $(0.004)$ \\
\hline$x x z$ & ppp & yes & - & yes (0.007) & - \\
\hline$x z x$ & ppp & yes & - & yes $(3.0)$ & - \\
\hline$z x x$ & ppp & yes (but 0) & yes & - & yes $(0.48)$ \\
\hline$z z z$ & ppp & yes & yes & yes $(0.03)$ & - \\
\hline$y y x$ & ssp & - & - & yes $(0.005)$ & - \\
\hline$y y z$ & ssp & yes & - & yes $(0.005)$ & - \\
\hline$z y y$ & pss & yes (but 0) & yes & - & yes $(0.31)$ \\
\hline$x y y$ & pss & - & - & - & yes $(0.44)$ \\
\hline$y x y$ & sps & - & - & - & yes $(0.2)$ \\
\hline$y z y$ & sps & yes & - & yes $(2.0)$ & - \\
\hline
\end{tabular}

TABLE I. All 14 possible non-zero elements of $\chi^{(2)}$, grouped according to the polarization scheme in which they are probed.

to which the formulas usually devoted to surface terms may be applied. In particular, an equivalent surface nonlinear susceptibility of the bulk is defined as

$$
P_{\mathrm{i}}^{\mathrm{B}, \text { surf }}\left(\mathbf{q}_{\mathrm{vis}}, \mathbf{q}_{\mathrm{IR}}\right)=\chi_{i j k, \mathrm{bulk}}^{(2)}\left(\mathbf{q}_{\mathrm{vis}}, \mathbf{q}_{\mathrm{IR}}\right) E_{j, \mathrm{vis}} E_{k, \mathrm{IR}} .
$$

Comparing SFG emitted in reflection and transmission, in principle, enables the bulk and surface contributions to be separated. ${ }^{55}$ It has been demonstrated that part of this bulk nonlinear susceptibility may be transformed into a true surface nonlinear susceptibility $\chi_{i j k, \text { bulk,insep. }}^{(2)}$, making it experimentally indistinguishable from $\chi_{i j k, \text { surf }}^{(2)} \cdot{ }^{4,53}$

Both formulations have their unique advantages, the first one facilitating modeling of the dispersion of the nonlinear bulk coefficients, and the second one providing a more facile route for comparing and combining bulk and surface terms. However, at first sight they may seem 
incompatible because the formulas for the emitted field in the bulk have diverged (compare for example Refs. 43 and 4). We show in Appendix A that they are in fact equivalent; we may therefore directly express $\chi_{i j k \text {,bulk }}^{(2)}$ as a function of the $D_{i}$ and $\Delta_{i}$ coefficients to calculate the bulk contribution. Care must be taken when describing the separable and inseparable bulk nonlinear susceptibilities, as the separable part of this equivalent surface nonlinear susceptibility only formally behaves as a true surface nonlinear susceptibility as far as SFG emission is concerned, but essentially differs through its wavevector ( $\mathbf{q}_{\mathrm{vis}}, \mathbf{q}_{\mathrm{IR}}$ and $\Delta \mathbf{q}$ ) dependence. As a consequence, the usual symmetry considerations do not apply to this tensor and a total of 14 terms is expected, listed in Table I. As detailed in Appendix B, a subset of these coefficients are intrinsically inseparable from the $z x x$, $z y y$ and $z z z$ surface terms. In the following, we focus on the separable contributions (Eqs. B5-B8 and B12-B15).

Our goal is to tune the angle of incidence $\theta$ to separate the various contributions to the gold SFG response as a result of their specific angular dependence. Among the bulk separable terms of the equivalent nonlinear surface susceptibility, those that do not vary with $\theta$ behave exactly like a surface term in our experiment. Following our previous work, ${ }^{27}$ we have calculated them for both the free and bound electron populations of gold, and display their magnitudes in Fig. 2. We note that the most intense terms ( $x z x, y z y$ and $z z z)$ are the same for both electron populations (this is also true for all other terms except $x x z$, $y y z, z z x$ and $y y x)$ and vary by no more than a few percent over the considered angle range, as do $x x z$ and $y y z$. These five elements cannot be separated from surface contributions in our measurements (see Table I). Therefore, in order to estimate the most important terms among the nine remaining ones, we calculate the total effective contribution by summing free and bound electron terms (although their mutual weights may be debated ${ }^{28}$ ) and weighting them by the appropriate Fresnel coefficients (Fig. 3). Their relative magnitude is indicated in the last column of Table I.

Maximal compatibility between bulk and surface terms is ensured when all quantities involved (electric fields and polarizations) are defined in the same medium, chosen as the place where the nonlinearity effectively takes place. In the present case, we consider this location to be inside gold, at $z=0^{-}$. 

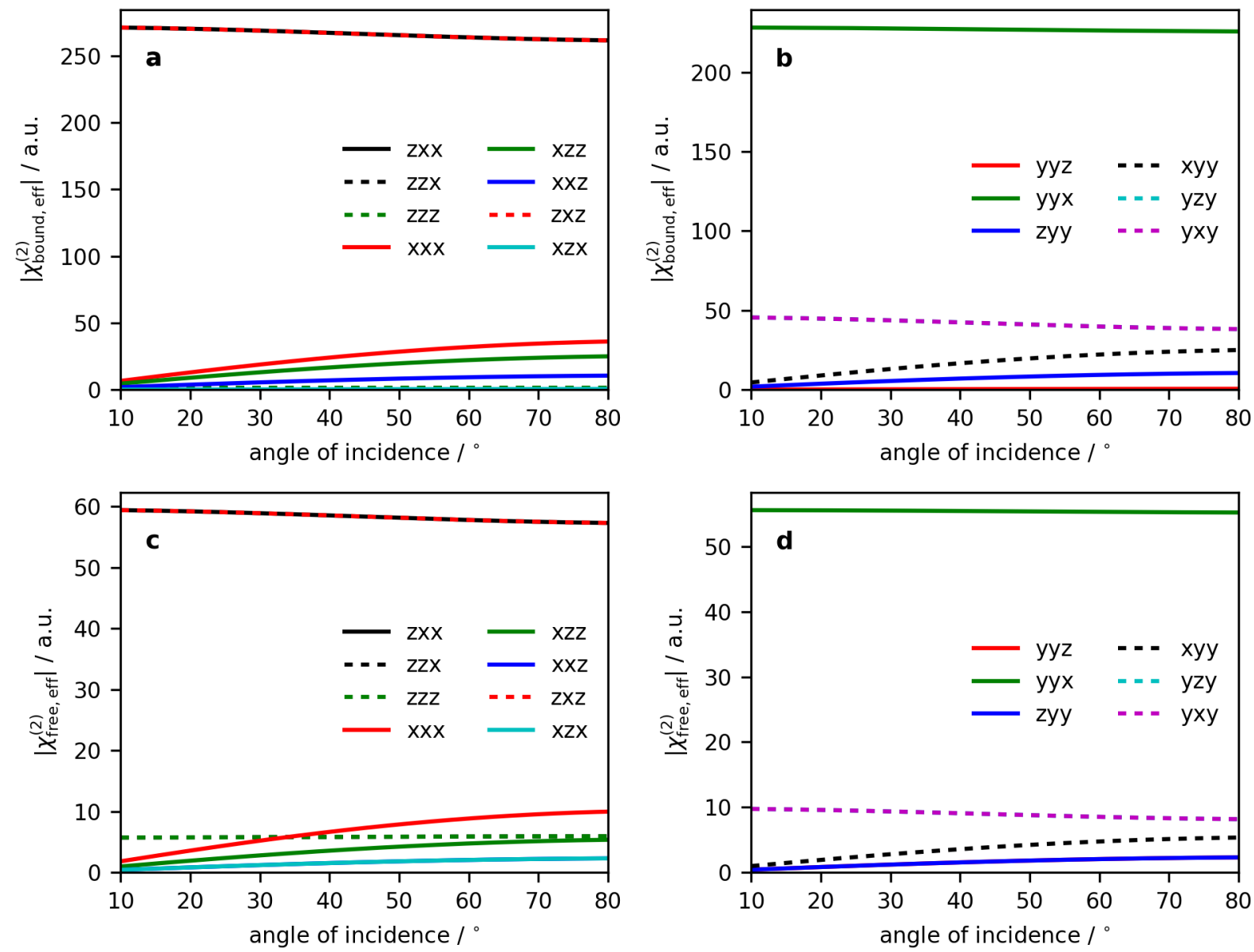

FIG. 2. Separable $\left|\chi_{\text {bulk }}^{(2)}\right|$ as a function of angle of incidence, dividing the contribution into that from the $(\mathrm{a}, \mathrm{b})$ the interband transition and $(\mathrm{c}, \mathrm{d})$ the free electron contribution.

\section{B. Local field considerations}

The intensity of the reflected SFG is obtained from

$$
I_{\mathrm{SFG}}=\frac{8 \pi^{3} \omega_{\mathrm{SFG}}^{2}}{c^{3} \cos ^{2} \theta_{\mathrm{SFG}}}\left|\chi_{\mathrm{eff}}^{(2)}\right|^{2} I_{\mathrm{vis}} I_{\mathrm{IR}}
$$

where the effective susceptibility is defined as

$$
\chi_{\mathrm{eff}}^{(2)}=L_{i i, \mathrm{SFG}} L_{j j, \mathrm{vis}} L_{k k, \mathrm{IR}} e_{i, \mathrm{SFG}} e_{j, \mathrm{vis}} e_{k, \mathrm{IR}}\left(\chi_{i j k, \mathrm{surf}}^{(2)}+\chi_{i j k, \mathrm{bulk}}^{(2)}\right) .
$$

This definition includes elements of the unit polarization vector

$$
\mathbf{e}=\left[\begin{array}{c} 
\pm \cos \theta \\
1 \\
\sin \theta
\end{array}\right]
$$



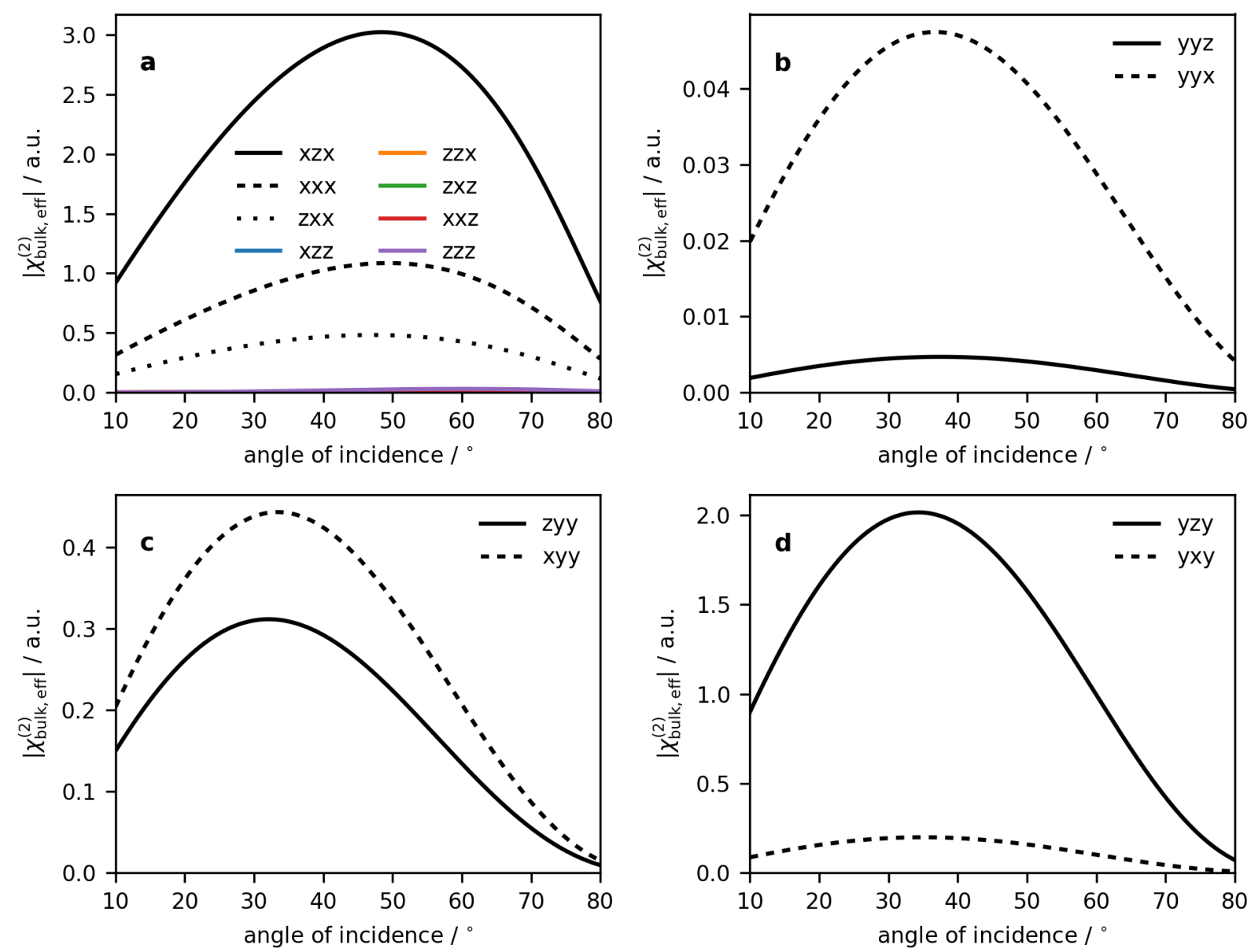

FIG. 3. Bulk effective $\chi^{(2)}$ elements as a function of angle of incidence for all four polarization schemes (a) PPP, (b) SSP, (c) PSS, and (d) SPS. The $x z z, z z x, z x z, x x z$, and $z z z$ elements that contribute to PPP are too small to be seen in comparison to $x z x, x x x, z x x$.

and local field correction tensor

$$
\mathbf{L}=\left[\begin{array}{ccc}
1-r_{p} & 0 & 0 \\
0 & 1+r_{s} & 0 \\
0 & 0 & \left(1+r_{p}\right) \frac{1}{n^{2}}
\end{array}\right]
$$

with $r_{p}$ and $r_{s}$ the familiar Fresnel reflection coefficients

$$
\begin{aligned}
& r_{p}=\frac{n \cos \theta-\cos \theta^{\mathrm{T}}}{\cos \theta^{\mathrm{T}}+n \cos \theta} \\
& r_{s}=\frac{\cos \theta-n \cos \theta^{\mathrm{T}}}{\cos \theta+n \cos \theta^{\mathrm{T}}}
\end{aligned}
$$

where the refracted angle $\theta^{\mathrm{T}}$ is obtained from Snell's law. As a reminder, we have assumed the refractive index is unity for air, and $n$ refers to the complex refractive index of bulk 

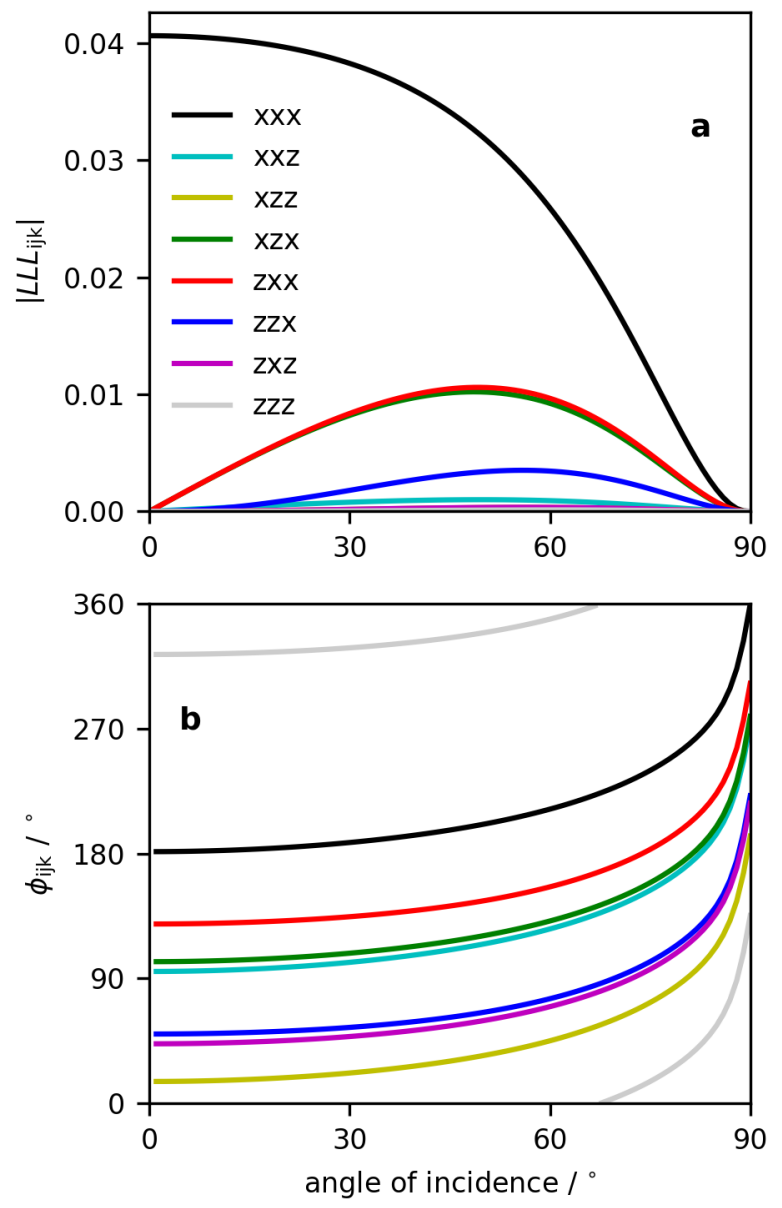

FIG. 4. A comparison angle-dependent (a) magnitude and (b) phase of the local field correction factors for the air-gold interface.

gold in all of our expressions. In our experimental geometry (Fig. 1), positive values of $e_{x}$ are used to describe in the incoming visible and IR beams; a negative value of $e_{x}$ is used in the case of the reflected SFG beam. For the vibrationally non-resonant response of gold, the form of $L_{z z}$ indicates that we treat the signal as if it originates from the bulk gold just below the surface (at $z=0^{-}$).

The relative magnitude and angle dependence of the $\mathbf{L}$ factors applicable to the ppp polarization scheme is shown in Fig. 4 for the case air-gold interface. In the case where the refractive index of the visible and SFG beams is similar, and the angle of the reflected SFG is close to the angle of incident visible beam (they are equal in our collinear geometry) we expect $L_{x x, \mathrm{SFG}} L_{z z_{\mathrm{vis}}}=L_{z z, \mathrm{SFG}} L_{x x, \mathrm{vis}}$. 


\section{EXPERIMENTAL METHODS}

Our $10 \mathrm{~Hz}$ wavelength-scanning SFG system has been described in Ref. 63 and the phasesensitive detection and analyis has been illustrated in detail in Refs. 64 and 65 . In the present experiments, a collinear configuration of a $532 \mathrm{~nm}$ beam (150 $\mu \mathrm{J}$ per pulse) and mid-IR beam at $2800 \mathrm{~cm}^{-1}\left(200 \mu \mathrm{J}\right.$ per pulse, $4 \mathrm{~cm}^{-1}$ bandwidth $)$ are incident at varying angles with a computer-controlled sample stage and detector arm. The external SFG field (local oscillator, LO) for the heterodyne measurements is generated in transmission from a piece of $50 \mu \mathrm{m} y$-cut $\alpha$-quartz with its optical axis rotated a few degrees from the plane of the incident polarization. Although this provided a low intensity LO, it also minimizes the change in polarization of all beams on account of the birefringence of the $y$-cut quartz. Data are collected by rotating a $1 \mathrm{~mm}$ fused silica phase shifting unit between the LO and sample at each incident angle of interest. The generated signal is a temporal interferogram along the phase-shifting axis and a spectral interferogram along the IR frequency axis. ${ }^{64}$ Our phase calibration utilizes a piece of $z$-cut $\alpha$-quartz as the reference sample, ${ }^{66}$ whose phase has been previously determined in the ssp polarization scheme. ${ }^{64,65}$ Samples consisted of vapour deposited $(100 \mathrm{~nm})$ gold over a $5 \mathrm{~nm}$ Cr adhesion layer on a glass substrate (EMF, Ithaca NY), cleaned with acetone and anhydrous ethanol prior to measurements. In the ppp polarization scheme, the phase of the $z$-cut $\alpha$-quartz is determined by following the derivation of Ref. 67 together with our knowledge of the already determined phase of the $z$-cut $\alpha$-quartz in the ssp polarization. We also assume that chiral elements of the nonlinear susceptibility do not contribute significantly to the SFG response of the bulk phase of the quartz, and that surface response is negligible when the plane of incidence is close to the crystallographic axis. ${ }^{68}$

\section{RESULTS}

We introduce the notation that we will use in the remaining sections. $\chi^{(2)}$ originating from the surface dipolar response is abbreviated $\chi_{\text {surf }}^{(2)}$ (first column in Table I). The term $\chi_{\text {comb.surf }}^{(2)}$ is used when describing the sum of all responses independent of the incident angle (first three

columns). $\chi_{\text {bulk }}^{(2)}$ is used to refer to the bulk response with incident angle dependence (fourth column). The only exception is the $x x z$ element probed in the ppp polarization scheme, 
where the $\chi_{x x z \text {,bulk }}^{(2)}$ refers to both the angle-dependent and angle-independent contribution. Any exceptions to this convention will be explicitly stated.

\section{A. ssp polarization scheme}

We have demonstrated that the separable bulk contributions are negligible in the ssp polarization scheme. We drop the subscripts (SFG, vis, IR) from elements of $\mathbf{L}$ and $\mathbf{e}$ as their order and designation is understood. We then arrive at the expression

$$
\begin{aligned}
\chi_{\mathrm{ssp}}^{(2)}= & L_{y y} e_{y} L_{y y} e_{y} L_{x x} e_{x} \chi_{y y x, \mathrm{bulk}}^{(2)}+L_{y y} e_{y} L_{y y} e_{y} L_{z z} e_{z} \chi_{y y z, \mathrm{surf}}^{(2)} \\
& \quad+L_{y y} e_{y} L_{y y} e_{y} L_{z z} e_{z} \chi_{y y z, \mathrm{bulk}}^{(2)} \\
\approx & L_{y y} e_{y} L_{y y} e_{y} L_{z z} e_{z} \chi_{y y z, \text { surf }}^{(2)}
\end{aligned}
$$

where the surface contribution dominates in ssp.

Results of homodyne measurements show the magnitude of the effective susceptibility $\chi_{\mathrm{ssp}}^{(2)}$ in Fig. 5 (squares) and fit to our model of the local field correction factors (dashed line). Although it was possible to collect gold ssp data at $532 \mathrm{~nm}$ with acceptable signal-to-noise, this is not necessarily the case for all metals or at different wavelengths in the visible or nearinfrared as a result of the frequency- and angle-dependent local field correction factors, the elements of $\mathbf{L}$ that appear in Eq. 11. Upon dividing by these factors, we obtain $\chi_{y y z}^{(2)}$ (circles) that is independent of the angle of incidence (solid line) as expected. This also illustrates our experimental accuracy in maintaining overlap of the beams and alignment into the detector as the sample is rotated by $30^{\circ}$. The fact that we can remove practically all angle dependent contributions in this treatment also justifies the assumption that $\chi_{y y x \text {,bulk }}^{(2)} \approx \chi_{y y z, \text { bulk }}^{(2)} \approx 0$. We have also measured the phase of $\chi_{y y z \text {,surf }}^{(2)}$ in an ssp heterodyne experiment and determined it to be $65 \pm 2^{\circ}$ at $2800 \mathrm{~cm}^{-1}$.

\section{B. ppp polarization scheme}

We now turn to our main interest in treating multiple angle of incidence ppp data. Here there are eight elements of $\chi^{(2)}$ that can contribute. In the case of our surface with azimuthal symmetry $\left(C_{\infty v}\right)$, there are only four non-zero elements that contribute to the surface dipolar response. In the bulk, all eight elements contribute to the overall nonlinear signal. The net 


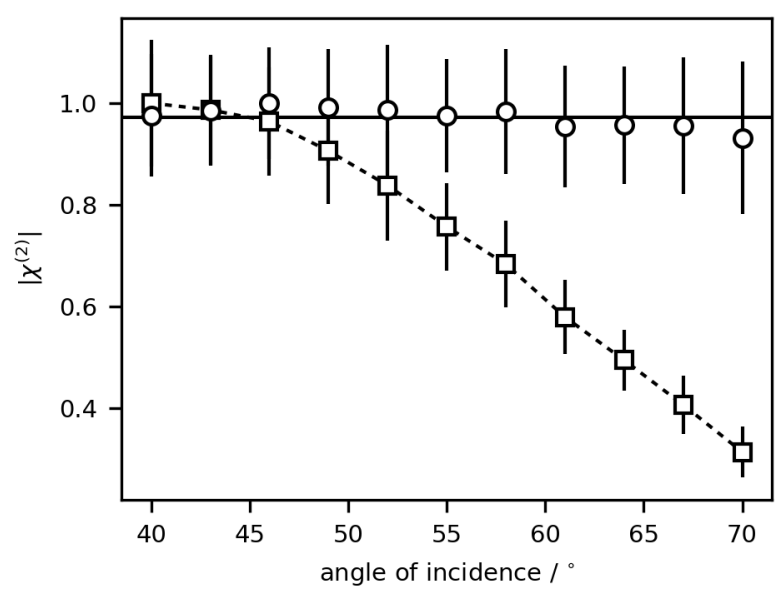

FIG. 5. Homodyne (intensity) measurements of the magnitude of the effective susceptibility $\left|\chi_{\text {ssp }}^{(2)}\right|$ at the air-gold interface as a function of angle (squares). As the data fits well to our model of $\left|L_{y y} e_{y} L_{y y} e_{y} L_{z z} e_{z}\right|$ (dashed line), dividing the data by the local field corrections and unit polarization vectors produces an essentially angle-independent result (circles). Error bars indicate one standard deviation about the mean. The errors are small for large angles due to the higher signal to noise ratio.

result is

$$
\begin{aligned}
\chi_{\mathrm{ppp}}^{(2)}= & L_{x x} e_{x} L_{x x} e_{x} L_{z z} e_{z} \chi_{x x z, \text { comb.surf }}^{(2)}+L_{x x} e_{x} L_{z z} e_{z} L_{x x} e_{x} \chi_{x z x, \text { comb.surf }}^{(2)} \\
& +L_{z z} e_{z} L_{x x} e_{x} L_{x x} e_{x} \chi_{z x x, \text { comb.surf }}^{(2)}+L_{z z} e_{z} L_{z z} e_{z} L_{z z} e_{z} \chi_{z z z, \text { comb.surf }}^{(2)} \\
& +L_{x x} e_{x} L_{x x} e_{x} L_{x x} e_{x} \chi_{x x x, \text { bulk }}^{(2)}+L_{x x} e_{x} L_{x x} e_{x} L_{z z} e_{z} \chi_{x x z, \text { bulk }}^{(2)} \\
& +L_{x x} e_{x} L_{z z} e_{z} z L_{x x} e_{x} \chi_{x z x, \text { bulk }}^{(2)}+L_{x x} e_{x} L_{z z} e_{z} z L_{z z} e_{z} \chi_{x z z, \text { bulk }}^{(2)} \\
& +L_{z z} e_{z} L_{x x} e_{x} L_{x x} e_{x} \chi_{z x x, \text { bulk }}^{(2)}+L_{z z} e_{z} L_{x x} e_{x} L_{z z} e_{z} \chi_{z x z, \text { bulk }}^{(2)} \\
& +L_{z z} e_{z} L_{z z} e_{z} L_{x x} e_{x} \chi_{z z x, \text { bulk }}^{(2)}+L_{z z} e_{z} L_{z z} e_{z} L_{z z} e_{z} \chi_{z z z, \text { bulk }}^{(2)}
\end{aligned}
$$

Some assumptions can be made in order to simplify the above expression. Given the relative size and angle dependence of the $\chi_{\text {bulk }}^{(2)}$ terms, some can be merged (indistinguishable in their angle-dependence) or dropped (negligible magnitude). The model has revealed that all $x z z$, $z z x$, and $z x z$ bulk terms are found to be insignificant relative to the rest of the bulk response. We therefore make the approximation that those three terms can be excluded, given that the they do not have corresponding non-zero dipolar terms.

We next examine the angle dependence of the $\chi_{\text {bulk }}^{(2)}$. From the electron gas model, we can see that only the bulk $x x x$ and $z x x$ terms are not constant with respect to the angle of 
incidence. Thus, we can treat the $x x z, x z x$ and $z z z$ bulk terms as complex-valued constants and merge them with their respective dipolar response, with the exception of the $z x x$ tensor element. According to the model, $z x x$ should not contribute to the surface dipolar response. However, the bulk contribution that is not separable from the overall surface response cannot be ignored. Thus, the overall surface response of the $z x x$ tensor element is not zero, as it originates from the bulk. This term in our expression is merged with its respective bulk term given that we are interested in the extraction of the surface tensor terms. Finally, we arrive at the simplified expression of $\chi_{\mathrm{ppp}}^{(2)}$, where each of the $\chi_{i j k, \text { comb.surf }}^{(2)}$ can be treated as a complex-valued constant

$$
\begin{aligned}
\chi_{\mathrm{ppp}}^{(2)} \approx & L_{x x} e_{x} L_{x x} e_{x} L_{z z} e_{z} \chi_{x x z, \text { comb.surf }}^{(2)}+L_{x x} e_{x} L_{z z} e_{z} L_{x x} e_{x} \chi_{x z x, \text { comb.surf }}^{(2)} \\
& +L_{z z} e_{z} L_{z z} e_{z} L_{z z} e_{z} \chi_{z z z, \text { comb.surf }}^{(2)}+L_{x x} e_{x} L_{x x} e_{x} L_{x x} e_{x} \chi_{x x x, \text { bulk }}^{(2)} \\
& +L_{z z} e_{z} L_{x x} e_{x} L_{x x} e_{x} \chi_{z x x, \text { bulk }}^{(2)}
\end{aligned}
$$

The ppp experiment is performed in the same way as for the ssp polarization scheme, except that we need to take into account that the phase of the LO is shifted by $180^{\circ}$ upon reflection from the $z$-cut quartz on either side of the Brewster angle. After the interference fringes have been suitably processed, ${ }^{64,65}$ we arrive at the results shown with points in Fig. 6.

To further simplify the expression of $\chi_{\mathrm{ppp}}^{(2)}$, we can represent the two angle dependent bulk elements $(z x x$ and $x x x)$ as well as their respective local field factors as a set of second-order polynomials for their real and imaginary components. This approximation is justified by first examining the linear combination of the $z x x$ and $x x x$ local field factors with the model value of $\chi^{(2)}$ (numbers in parenthesis in Table I). We then fit the line shape for its real and imaginary components with separate second-order polynomial function and found it is reasonable in this approach to capture the line shape in the experimental range of $40-70^{\circ}$. Thus, the $\chi_{\mathrm{ppp}}^{(2)}$ expression for the multi-angle of incidence fitting becomes

$$
\begin{aligned}
\chi_{\mathrm{ppp}}^{(2)} \approx & (\text { LLLeee })_{x x z}(\theta) \chi_{x x z, \text { surf }+ \text { bulk }}^{(2)}+(\text { LLLeee })_{x x z}(\theta) \chi_{x z x, \text { surf }+ \text { bulk }}^{(2)} \\
& +(\text { LLLeee })_{z z z}(\theta) \chi_{z z z, \text { surf }+ \text { bulk }}^{(2)}+a \theta^{2}+b \theta+c
\end{aligned}
$$

where $(L L L e e e)_{i j k}$ is the product of the local field factors and unit polarization vectors for all three fields (SFG, visible, IR) for a given tensor elements. The coefficients $a, b$ and $c$ encompass the linear combination of the product of the local field factors, unit polarization 

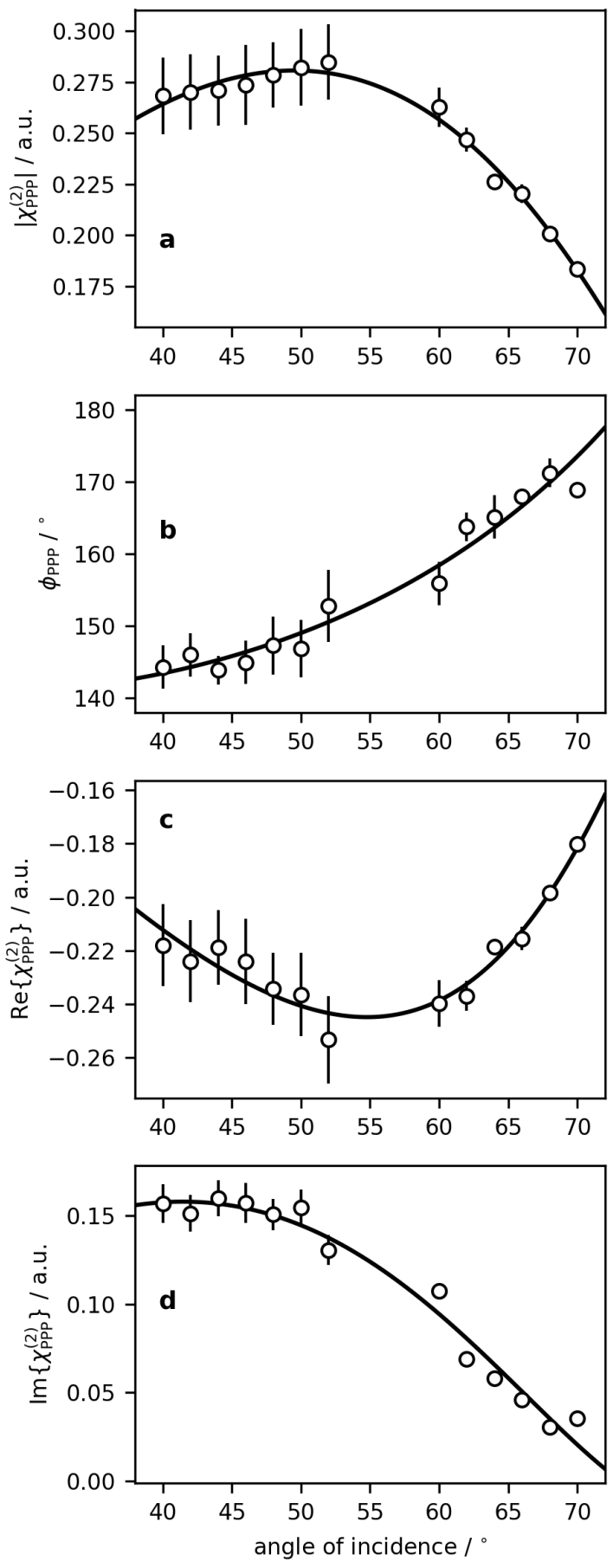

FIG. 6. Experimental results (points) from the heterodyne measurements in the ppp polarization scheme displaying the (a) magnitude, (b) phase, (c) real, and (d) imaginary components of $\chi_{\mathrm{ppp}}^{(2)}$. A fit of this data to Eq. 14 is shown with the lines in each panel. Error bars indicate one standard deviation about the mean. 
vectors, and $\chi_{\text {bulk }}^{(2)}$ for the $z x x$ and $x x x$ components. As a result of the angle dependence of $\mathbf{L}$, (see Fig. 4) and the two angle dependent $\chi^{(2)}$ terms $(z x x$ and $x x x$ ), we can solve for all the surface tensor elements. From our fitting, we find that $|x z x / z z z|=0.39 \pm 0.02$, $|x x z / z z z|=1.11 \pm 0.06, \phi_{x z x}=-146 \pm 3^{\circ}, \phi_{x x z}=62 \pm 3^{\circ}$, and $\phi_{z z z}=83 \pm 3^{\circ}$.

\section{DISCUSSION}

Before bench-marking our experimental fitting results, it is important to first understand what each of the simplified tensor elements represents; the three elements denoted as 'surf+bulk' are especially interesting. In the case of the $x z x$ component, the bulk response is significant relative to the overall bulk contribution. Thus, the $x z x$ component is the combination of the dipolar surface contribution as well as the quadrupolar bulk contribution. For the case of the $x x z$ and $z z z$ elements, their quadrupolar bulk contribution are insignificant relative to the rest. Thus, as an approximation, we can treat the two tensor elements as the dipolar surface response only, where $\chi_{i j k, \text { comb.surf }}^{(2)} \approx \chi_{i j k \text {,surf }}^{(2)}$.

There are two tests we can perform in order to validate our results. As our gold surface has $C_{\infty v}$ symmetry, we know that $\chi_{x x z, \text { surf }}^{(2)}=\chi_{y y z, \text { surf }}^{(2)}$ for both the magnitude and phase of these tensor elements. Our first check is therefore to compare the result $\phi_{x x z, \text { surf }}=62 \pm 3^{\circ}$ obtained in our deconstruction of the ppp data to our result of $\phi_{y y z \text {,surf }}=65 \pm 2^{\circ}$ measured directly in the ssp heterodyne experiment; we find them to be in agreement. The good match also validates our model result that the relative contribution of $\chi_{x x z \text {,bulk }}^{(2)}$ is predicted to be small. The second check is a comparison between experimental ppp/ssp ratio (points in Fig. 7) and the predictions of our model in extracting the magnitude of all tensor elements contributing to the ppp signal. If we take the magnitude of $\chi_{x x z}^{(2)}$ from the ppp fit and multiply by the magnitude of $L_{y y z}$ we should reproduce the ssp data according to

$$
\begin{aligned}
& \left|\chi_{\mathrm{ssp}}^{(2)}\right| \approx\left|L_{y y} e_{y} L_{y y} e_{y} L_{z z} e_{z} \chi_{y y z, \mathrm{surf}}^{(2)}\right| \\
& =\left|L_{y y} e_{y} L_{y y} e_{y} L_{z z} e_{z} \chi_{x x z, \text { surf }}^{(2)}\right| .
\end{aligned}
$$

The predicted ratio is plotted with the solid line in Fig. 7. The excellent agreement further validates the decomposition of $\chi^{(2)}$ tensor elements with our multi-angle ppp experiment. Note that, although incorporation of the low-intensity ssp data has introduced large error bars at high angles, this is not a fit (no adjustable parameters), merely a comparison of two independent results. 


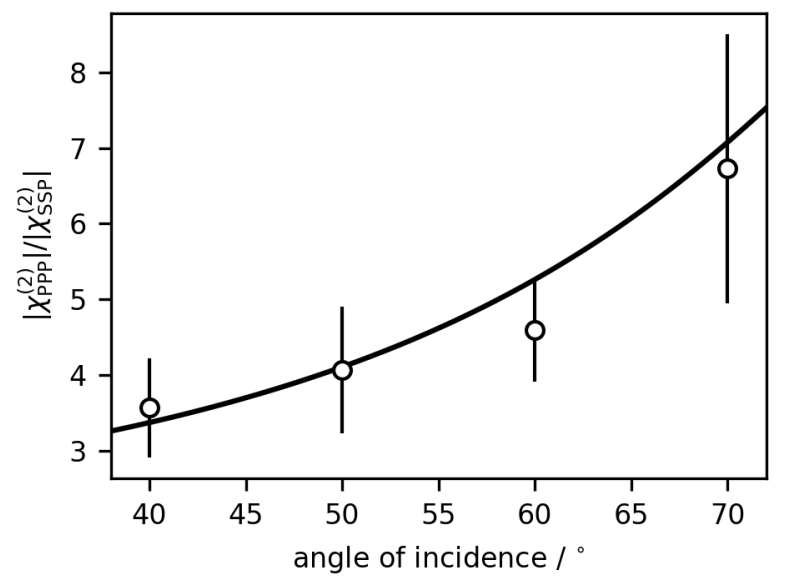

FIG. 7. Comparison of the ratio of the magnitudes of the effective susceptibilities measured in separate ppp and ssp homodyne experiments (points), to that calculated from the ppp multi-angle heterodyne data (line), demonstrating the equivalence expressed in Eq. 15. Error bars represent one standard deviation about the mean.

The decomposition of the three surface $\chi^{(2)}$ elements with their respective local field factors and the total bulk contribution is presented in Fig. 8. Without accounting for local field effects, the relative size of the three surface tensor elements follows the order of $x x z>z z z>x z x$. In comparison, it is found that the experimental gold $\chi_{\mathrm{ppp}}^{(2)}$ response is dominated by the effective surface $x z x$ term followed by the $x x z$ term then the $z z z$ term. This is due to the local field factor essentially acts as a relative weighting factor to its respective tensor element. More interestingly, the total contribution of the bulk response is significantly smaller than the rest of the surface response in Fig. 8. Due to the screening of the $z$-component inside the gold when crossing the interface, components parallel to the surface are much larger than the perpendicular ones. This result is in agreement with Refs. 69 and 70, given that the surface $\chi^{(2)}$ elements contain both the isotropic and inseparable bulk contributions.

From the model result in Table I, there should be a significant contribution from the $x x x$ and $z x x$ elements relative to the total bulk response. Furthermore, given the relative size of the $x x x$ and $z x x$ local field factors, one would expect that the total bulk contribution should be significant compared to the surface response. We conclude that the total separable bulk contribution is smaller than the surface and inseparable bulk response. This is in agreement 

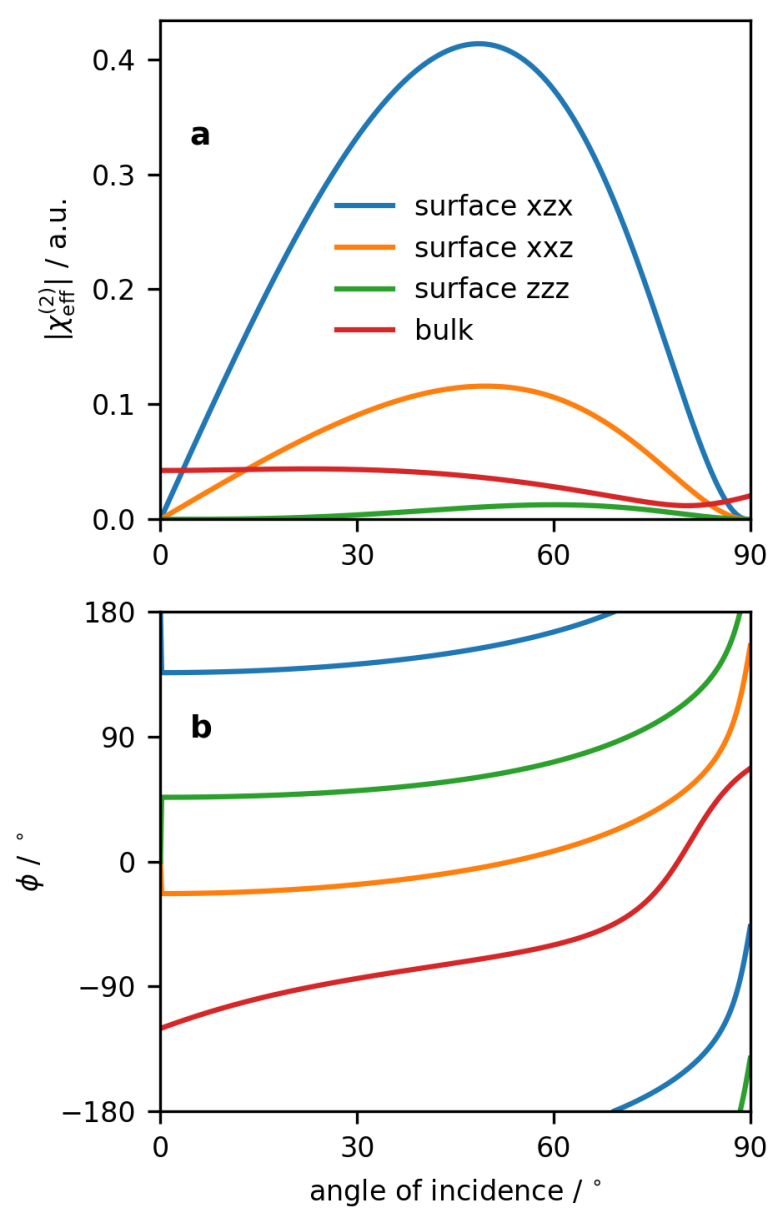

FIG. 8. Comparison of the (a) magnitude and (b) phase of the product of the susceptibility from the fitting and its respective local field factor

with what has been reported for other metals, in studies where oxidation or disordering decreases the nonlinear response. ${ }^{61}$

\section{CONCLUSIONS}

A heterodyne-detected multi-angle approach has been demonstrated to decompose the second-order nonlinear susceptibility measured in an SFG experiment in order to obtain the substrate optical properties. As a demonstration, we have performed a study of a planar gold surface that necessitates consideration of dipolar surface contributions as well as quadrupolar bulk contributions. This method is especially interesting and relevant in cases where only ppp experiments produce appreciable signals on account of surface selection 
rules. In the case where organic molecules are adsorbed on metal surfaces, this method has the potential to assist in separating substrate and adsorbate components of the nonlinear susceptibility tensor. While additional challenges remain in the case of vibrationally-resonant contributions, the work we have presented here provide the necessary theoretical framework, experimental techniques and results to embark on such studies.

\section{ACKNOWLEDGMENTS}

We thank the Natural Sciences and Engineering Research Council of Canada (NSERC) for support of this science with a Discovery Grant, in collaboration with Imperial Oil for a University Research Award and NSERC Collaborative Research and Discovery Grant. The collaboration between the University of Victoria and Université Paris-Saclay was supported by the French Embassy in Canada through the France-Canada Research Fund. Dr. Stanislav Konorov (Univeristy of Victoria, Department of Chemistry and Centre for Advanced Materials and Related Technologies (CAMTEC)) provided valuable advice and assistance with the lasers.

\section{Appendix A: Bulk SFG contribution transformed into a surface term}

We recall ${ }^{29}$ that, inside the bulk, the $\mathrm{SFG}$ beam propagates with a wavevector $\mathbf{q}_{\mathrm{SFG}}$, a dielectric function $\epsilon_{\mathrm{SFG}}$ and at an angle of incidence $\theta_{\mathrm{SFG}}^{\mathrm{T}}$, whereas the nonlinear polarization propagates with a wavevector $\mathbf{q}_{\mathrm{SFG}}^{S}=\mathbf{q}_{\mathrm{vis}}+\mathbf{q}_{\mathrm{IR}}$, associated to a dielectric function $\epsilon_{S}=$ $n_{S}^{2}=\epsilon_{\mathrm{SFG}}\left|\mathbf{q}_{\mathrm{SFG}}^{S}\right|^{2} /\left|\mathbf{q}_{\mathrm{SFG}}\right|^{2}$ at an angle of incidence $\theta_{\mathrm{SFG}}^{S}$. Starting from the original equations giving the amplitudes of the reflected SFG field as a function of the bulk polarization ${ }^{42}$

$$
\begin{array}{r}
E_{\mathrm{p}}\left(\omega_{\mathrm{SFG}}\right)=\frac{4 \pi}{n_{S} n_{\mathrm{SFG}}} \frac{1}{n_{\mathrm{SFG}} \cos \theta_{\mathrm{SFG}}+\cos \theta_{\mathrm{SFG}}^{T}}\left[P_{\|}^{\mathrm{B}} \sin \theta_{\mathrm{SFG}}\right. \\
\left.+P_{\perp}^{\mathrm{B}} \frac{\epsilon_{\mathrm{SFG}} n_{S} \cos \theta_{\mathrm{SFG}}^{\mathrm{S}}-\epsilon_{S} n_{\mathrm{SFG}} \cos \theta_{\mathrm{SFG}}^{\mathrm{T}}}{\epsilon_{\mathrm{SFG}}-\epsilon_{S}}\right]
\end{array}
$$

and

$$
E_{\mathrm{s}}\left(\omega_{\mathrm{SFG}}\right)=E_{\mathrm{y}}\left(\omega_{\mathrm{SFG}}\right)=\frac{4 \pi}{\epsilon_{\mathrm{SFG}}-\epsilon_{S}} \frac{n_{S} \cos \theta_{\mathrm{SFG}}^{\mathrm{S}}-n_{\mathrm{SFG}} \cos \theta_{\mathrm{SFG}}^{\mathrm{T}}}{n_{\mathrm{SFG}} \cos \theta_{\mathrm{SFG}}^{T}+\cos \theta_{\mathrm{SFG}}^{\mathrm{B}}} P_{y}
$$

where $P_{\|}^{\mathrm{B}}$ and $P_{\perp}^{\mathrm{B}}$ stand for the components of the bulk nonlinear polarization parallel and perpendicular to $\mathbf{q}_{\mathrm{SFG}}^{S}$, respectively, in the $(x, z)$ plane. Defining the wavevector mismatch 
in reflection $\left(\Delta q_{z}\right)_{R}=q_{\mathrm{vis}, \mathrm{z}}+q_{\mathrm{IR}, \mathrm{z}}+q_{\mathrm{SFG}, \mathrm{z}}$ and in transmission $\left(\Delta q_{z}\right)_{T}=q_{\mathrm{vis}, \mathrm{z}}+q_{\mathrm{IR}, \mathrm{z}}-q_{\mathrm{SFG}, \mathrm{z}}$, we use the following identities to transform Eq. A1 and A2

$$
\begin{aligned}
&\left(\mathbf{q}_{\mathrm{SFG}}^{S}\right)^{2}-\left(\mathbf{q}_{\mathrm{SFG}}\right)^{2}=\left(q_{\mathrm{SFG}, \mathrm{z}}^{S}\right)^{2}-\left(q_{\mathrm{SFG}, \mathrm{z}}\right)^{2} \\
&=\left(\Delta q_{z}\right)_{R}\left(\Delta q_{z}\right)_{T} \\
&=\frac{\omega_{\mathrm{SFG}}^{2}}{c^{2}}\left(\epsilon_{\mathrm{S}}-\epsilon_{3}\right) \\
&\left(\Delta q_{z}\right)_{R / T}=\frac{\omega_{\mathrm{SFG}}}{c}\left(n_{S} \cos \theta_{\mathrm{SFG}}^{\mathrm{S}} \pm n_{\mathrm{SFG}} \cos \theta_{\mathrm{SFG}}^{\mathrm{T}}\right) .
\end{aligned}
$$

For s-polarization, this leads to

$$
\begin{aligned}
E_{\mathrm{s}}\left(\omega_{\mathrm{SFG}}\right) & =\frac{4 i \pi \omega_{\mathrm{SFG}}}{c} \frac{1}{n_{\mathrm{SFG}} \cos \theta_{\mathrm{SFG}}^{T}+\cos \theta_{\mathrm{SFG}}} \frac{i P_{y}^{\mathrm{B}}}{\left(\Delta q_{z}\right)_{R}} \\
& =\frac{2 i \pi \omega_{\mathrm{SFG}}}{\cos \theta_{\mathrm{SFG}} c} L_{y y}\left(\omega_{\mathrm{SFG}}\right) \frac{i P_{y}^{\mathrm{B}}}{\left(\Delta q_{z}\right)_{R}}
\end{aligned}
$$

and for p-polarization we have

$$
\begin{aligned}
P_{\|}^{\mathrm{B}} & =P_{x}^{\mathrm{B}} \sin \theta_{\mathrm{SFG}}^{\mathrm{S}}-P_{\mathrm{z}}^{\mathrm{B}} \cos \theta_{\mathrm{SFG}}^{\mathrm{S}} \\
P_{\perp}^{\mathrm{B}} & =P_{x}^{\mathrm{B}} \cos \theta_{\mathrm{SFG}}^{\mathrm{S}}+P_{\mathrm{z}}^{\mathrm{B}} \sin \theta_{\mathrm{SFG}}^{\mathrm{S}} .
\end{aligned}
$$

Using

$$
\frac{\epsilon_{\mathrm{SFG}} n_{\mathrm{S}} \cos \theta_{\mathrm{SFG}}^{\mathrm{S}}-\epsilon_{\mathrm{S}} n_{\mathrm{SFG}} \cos \theta_{\mathrm{SFG}}^{\mathrm{T}}}{\epsilon_{\mathrm{SFG}}-\epsilon_{\mathrm{S}}}=n_{\mathrm{SFG}}\left(\cos \theta_{\mathrm{SFG}}^{T}-\frac{\omega_{\mathrm{SFG}}}{c} \frac{n_{\mathrm{SFG}}}{\left(\Delta q_{\mathrm{z}}\right)_{R}}\right)
$$

we get, for the $x$ projection,

$$
\begin{aligned}
& E_{\mathrm{p}, \mathrm{x}}\left(\omega_{\mathrm{SFG}}\right)= \frac{4 \pi}{n_{\mathrm{S}} n_{\mathrm{SFG}}} \frac{1}{n_{\mathrm{SFG}} \cos \theta_{\mathrm{SFG}}+\cos \theta_{\mathrm{SFG}}^{T}}\left[\sin \theta_{\mathrm{SFG}}^{\mathrm{S}} \sin \theta_{\mathrm{SFG}}\right. \\
&\left.+\cos \theta_{\mathrm{SFG}}^{\mathrm{S}} n_{\mathrm{SFG}}\left(\cos \theta_{\mathrm{SFG}}^{T}-\frac{\omega_{\mathrm{SFG}}}{c} \frac{n_{\mathrm{SFG}}}{\left(\Delta q_{\mathrm{z}}\right)_{R}}\right)\right] P_{\mathrm{x}}^{\mathrm{B}} .
\end{aligned}
$$

Using $\sin \theta_{\mathrm{SFG}}=n_{\mathrm{SFG}} \sin \theta_{\mathrm{SFG}}^{T}$ and

$$
\left(\Delta q_{z}\right)_{R} \cos \left(\theta_{\mathrm{SFG}}^{T}-\theta_{\mathrm{SFG}}^{S}\right)=\frac{\omega_{\mathrm{SFG}}}{c}\left[n_{\mathrm{SFG}} \cos \theta_{\mathrm{SFG}}^{S}+n_{S} \cos \theta_{\mathrm{SFG}}^{\mathrm{T}}\right]
$$

this simplifies into

$$
\begin{aligned}
E_{\mathrm{p}, \mathrm{x}}\left(\omega_{\mathrm{SFG}}\right) & =\frac{4 \pi \omega_{\mathrm{SFG}}}{c} \frac{\cos \theta_{\mathrm{SFG}}^{\mathrm{T}}}{n_{\mathrm{SFG}} \cos \theta_{\mathrm{SFG}}+\cos \theta_{\mathrm{SFG}}^{T}}\left[\frac{P_{x}^{\mathrm{B}}}{\left(\Delta q_{\mathrm{z}}\right)_{R}}\right] \\
& =-\frac{2 i \pi \omega_{\mathrm{SFG}}}{\cos \theta_{\mathrm{SFG}} c} L_{\mathrm{xx}}\left(\omega_{\mathrm{SFG}}\right) \cos \theta_{\mathrm{SFG}} \frac{i P_{\mathrm{x}}^{\mathrm{B}}}{\left(\Delta q_{\mathrm{z}}\right)_{R}} .
\end{aligned}
$$


For the $z$ projection, we have

$$
\begin{array}{r}
E_{\mathrm{p}, \mathrm{z}}\left(\omega_{\mathrm{SFG}}\right)=\frac{4 \pi}{n_{S} n_{\mathrm{SFG}}} \frac{1}{n_{\mathrm{SFG}} \cos \theta_{\mathrm{SFG}}+\cos \theta_{\mathrm{SFG}}^{T}}\left[-\cos \theta_{\mathrm{SFG}}^{S} \sin \theta_{\mathrm{SFG}}\right. \\
\left.+\sin \theta_{\mathrm{SFG}}^{S} n_{\mathrm{SFG}}\left(\cos \theta_{\mathrm{SFG}}^{T}-\frac{\omega_{\mathrm{SFG}}}{c} \frac{n_{\mathrm{SFG}}}{\left(\Delta q_{z}\right)_{R}}\right)\right] P_{z}^{\mathrm{B}},
\end{array}
$$

simplifying by use of

$$
\left(\Delta q_{z}\right)_{R} \sin \left(\theta_{\mathrm{SFG}}^{T}-\theta_{\mathrm{SFG}}^{S}\right)=\frac{\omega_{\mathrm{SFG}}}{c} \frac{\epsilon_{S}-\epsilon_{\mathrm{SFG}}}{n_{S} n_{\mathrm{SFG}}} \sin \theta_{\mathrm{SFG}}
$$

into

$$
\begin{aligned}
E_{\mathrm{p}, \mathrm{z}}\left(\omega_{\mathrm{SFG}}\right) & =-\frac{4 \pi \omega_{\mathrm{SFG}}}{c n_{\mathrm{SFG}}} \frac{1}{n_{\mathrm{SFG}} \cos \theta_{\mathrm{SFG}}+\cos \theta_{\mathrm{SFG}}^{T}} \frac{P_{z}^{\mathrm{B}}}{\left(\Delta q_{z}\right)_{R}} \sin \theta_{\mathrm{SFG}} \\
& =\frac{2 i \pi \omega_{\mathrm{SFG}}}{c \cos \theta_{\mathrm{SFG}}} L_{z z}\left(\omega_{\mathrm{SFG}}\right) \sin \theta_{\mathrm{SFG}} \frac{i P_{z}^{\mathrm{B}}}{\left(\Delta q_{z}\right)_{R}} .
\end{aligned}
$$

Equations A5, A10 and A13 are formally identical to the decomposition of the electric field emitted at the interface by an equivalent surface polarization ${ }^{41}$ defined by Eq. 4 .

\section{Appendix B: Separable and inseparable bulk terms}

From Eq. 3, 4 and 5, and using Eq. 1, we get for the 14 components of the equivalent surface nonlinear susceptibility:

$$
\begin{gathered}
\chi_{x x x, \text { bulk }}^{(2)}=-\frac{1}{\left(\Delta q_{z}\right)_{R}}\left[D_{\mathrm{vis}} q_{\mathrm{vis}, x}+D_{\mathrm{IR}} q_{\mathrm{IR}, x}+\Delta_{\mathrm{vis}} q_{\mathrm{vis}, x}+\Delta_{\mathrm{IR}} q_{\mathrm{IR}, x}\right] \\
\chi_{x z z, \mathrm{bulk}}^{(2)}=\chi_{x y y, \text { bulk }}^{(2)}=-\frac{1}{\left(\Delta q_{z}\right)_{R}}\left[D_{\mathrm{vis}} q_{\mathrm{vis}, x}+D_{\mathrm{IR}} q_{\mathrm{IR}, x}\right] \\
\chi_{z x x, \text { bulk }}^{(2)}=\chi_{z y y, \text { bulk }}^{(2)}=\frac{1}{\left(\Delta q_{z}\right)_{R}}\left[D_{\mathrm{vis}} q_{\mathrm{vis}, z}+D_{\mathrm{IR}} q_{\mathrm{IR}, z}\right] \\
\chi_{z z z, \mathrm{bulk}}^{(2)}=\frac{1}{\left(\Delta q_{z}\right)_{R}}\left[D_{\mathrm{vis}} q_{\mathrm{vis}, z}+D_{\mathrm{IR}} q_{\mathrm{IR}, z}+\Delta_{\mathrm{vis}} q_{\mathrm{vis}, z}+\Delta_{\mathrm{IR}} q_{\mathrm{IR}, z}\right] \\
\chi_{x x z, \text { bulk }}^{(2)}=\chi_{y y z, \mathrm{bulk}}^{(2)}=\frac{1}{\left(\Delta q_{z}\right)_{R}}\left[\Delta_{\mathrm{vis}} q_{\mathrm{vis}, z}\right] \\
\chi_{x z x, \mathrm{bulk}}^{(2)}=\chi_{y z y, \mathrm{bulk}}^{(2)}=\frac{1}{\left(\Delta q_{z}\right)_{R}}\left[\Delta_{\mathrm{IR}} q_{\mathrm{IR}, z}\right] \\
\chi_{z z x, \text { bulk }}^{(2)}=\chi_{y y x, \text { bulk }}^{(2)}=-\frac{1}{\left(\Delta q_{z}\right)_{R}}\left[\Delta_{\mathrm{vis}} q_{\mathrm{vis}, x}\right] \\
\chi_{z x z, \mathrm{bulk}}^{(2)}=\chi_{y x y, \text { bulk }}^{(2)}=-\frac{1}{\left(\Delta q_{z}\right)_{R}}\left[\Delta_{\mathrm{IR}} q_{\mathrm{IR}, x}\right]
\end{gathered}
$$


It is known ${ }^{41}$ that only the transverse part of the bulk nonlinear polarization emits field at the SFG frequency, the transversality being related to its wavevector $\mathbf{q}_{\mathrm{SFG}}$. We rewrite Eq. 3 as

$$
\begin{aligned}
-i \mathbf{P}^{\mathbf{B}}\left(\mathbf{q}_{\mathrm{vis}}, \mathbf{q}_{\mathrm{IR}}\right) & =\left[\frac{D_{\mathrm{vis}}+D_{\mathrm{IR}}}{2}\left(\mathbf{q}_{\mathrm{vis}}+\mathbf{q}_{\mathrm{IR}}\right)\right. \\
& \left.+\frac{D_{\mathrm{vis}}-D_{\mathrm{IR}}}{2}\left(\mathbf{q}_{\mathrm{vis}}-\mathbf{q}_{\mathrm{IR}}\right)\right]\left(\mathbf{E}_{\mathrm{vis}} \cdot \mathbf{E}_{\mathrm{IR}}\right) \\
& +\Delta_{\mathrm{vis}}\left(\mathbf{q}_{\mathrm{vis}} \cdot \mathbf{E}_{\mathrm{IR}}\right) \mathbf{E}_{\mathrm{vis}}+\Delta_{\mathrm{IR}}\left(\mathbf{q}_{\mathrm{IR}} \cdot \mathbf{E}_{\mathrm{vis}}\right) \mathbf{E}_{\mathrm{IR}}
\end{aligned}
$$

We may, without modifying the emitted fields, subtract from the first term a non-radiative contribution $\left(D_{\mathrm{vis}}+D_{\mathrm{IR}}\right) \mathbf{q}_{\mathrm{SFG}}\left(\mathbf{E}_{\mathrm{vis}} \cdot \mathbf{E}_{\mathrm{IR}}\right) / 2$, so that it now contributes to the bulk nonlinear polarization as

$$
-i \frac{D_{\mathrm{vis}}+D_{\mathrm{IR}}}{2}\left(\Delta q_{z}\right)_{R}\left(\mathbf{E}_{\mathrm{vis}} \cdot \mathbf{E}_{\mathrm{IR}}\right) \hat{z}
$$

and to the equivalent surface polarization as

$$
\mathbf{P}^{\mathrm{B} \text {,surf,insep. }}=\frac{D_{\mathrm{vis}}+D_{\mathrm{IR}}}{2}\left(\mathbf{E}_{\mathrm{vis}} \cdot \mathbf{E}_{\mathrm{IR}}\right) \hat{z} .
$$

This part of the equivalent surface polarization amounts to adding the constant value $\left(D_{\text {vis }}+\right.$ $\left.D_{\text {IR }}\right) / 2$ to the surface coefficients $z x x, z y y$ and $z z z$. As it does not depend on any wavevector anymore, it is not possible to experimentally discriminate it from pure surface terms ${ }^{60}$, and is named inseparable bulk. ${ }^{55}$ On the contrary, the separable bulk terms of the equivalent surface nonlinear polarization follow, modifying Eqs. B1-B4 to obtain

$$
\begin{array}{r}
\chi_{x x x, \text { bulk,sep. }}^{(2)}=-\frac{1}{\left(\Delta q_{z}\right)_{R}}\left[\frac{D_{\mathrm{vis}}-D_{\mathrm{IR}}}{2}\left(q_{\mathrm{vis}, x}-q_{\mathrm{IR}, x}\right)+\Delta_{\mathrm{vis}} q_{\mathrm{vis}, x}+\Delta_{\mathrm{IR}} q_{\mathrm{IR}, x}\right] \\
\begin{aligned}
\chi_{x z z, \text { bulk,sep. }}^{(2)} & =\chi_{x y y, \text { bulk,sep. }}^{(2)} \\
= & -\frac{1}{\left(\Delta q_{z}\right)_{R}}\left[\frac{D_{\mathrm{vis}}-D_{\mathrm{IR}}}{2}\left(q_{\mathrm{vis}, x}-q_{\mathrm{IR}, x}\right)\right] \\
\chi_{z x x, \text { bulk,sep. }}^{(2)} & =\chi_{z y y, \text { bulk,sep. }}^{(2)} \\
& =\frac{1}{\left(\Delta q_{z}\right)_{R}}\left[\frac{D_{\mathrm{vis}}-D_{\mathrm{IR}}}{2}\left(q_{\mathrm{vis}, z}-q_{\mathrm{IR}, z}\right)\right] \\
\chi_{z z z, \text { bulk,sep. }}^{(2)}= & \frac{1}{\left(\Delta q_{z}\right)_{R}}\left[\frac{D_{\mathrm{vis}}-D_{\mathrm{IR}}}{2}\left(q_{\mathrm{vis}, z}-q_{\mathrm{IR}, z}\right)+\Delta_{\mathrm{vis}} q_{\mathrm{vis}, z}+\Delta_{\mathrm{IR}} q_{\mathrm{IR}, z}\right]
\end{aligned}
\end{array}
$$

while the eight others (Eq. B5-B8) remain unchanged. 


\section{REFERENCES}

${ }^{1}$ Y. R. Shen, in Proc. Int. School of Physics, Ernico Fermi, edited by T. W. Hansch and M. Inguscio (North Holland, Amsterdam, 1994) pp. 139-165.

${ }^{2}$ A. Morita, Theory of Sum Frequency Generation Spectroscopy (Springer, Singapore, 2018).

${ }^{3}$ P. B. Miranda and Y. R. Shen, J. Phys. Chem. B 103, 3292 (1999).

${ }^{4}$ Y. R. Shen, J. Phys. Chem. C 116, 15505 (2012).

${ }^{5}$ R. W. Boyd, Nonlinear Optics, 2nd ed. (Academic Press, San Diego, 2003).

${ }^{6}$ H.-F. Wang, W. Gan, R. Lu, Y. Rao, and B. H. Wu, Int. Rev. Phys. Chem. 24, 191 (2005).

${ }^{7}$ R. Lu, W. Gan, and H. Wang, Chin. Sci. Bull. 48, 2183 (2003).

${ }^{8}$ W. Gan, B.-h. Wu, H. Chen, Y. Guo, and H. Wang, Chem. Phys. Letts. 406, 467 (2005).

${ }^{9}$ H. Chen, W. Gan, B.-H. Wu, D. Wu, Z. Zhang, and H.-F. Wang, Chem. Phys. Lett. 408, $284(2005)$.

${ }^{10}$ S. A. Hall, K. C. Jena, P. A. Covert, S. Roy, T. G. Trudeau, and D. K. Hore, J. Phys. Chem. B 118, 5617 (2014).

${ }^{11}$ K.-K. Hung, U. Stege, and D. K. Hore, Appl. Spectrosc. Rev. 50, 351 (2015).

${ }^{12}$ H. Wu, W. Zhang, W. Gan, Z. Cui, and H. F. Wang, J. Chem. Phys. 125, 133203 (2006).

${ }^{13}$ K. Jena, K.-K. Hung, T. Schwantje, and D. K. Hore, J. Chem. Phys. 135, 044704 (2011).

${ }^{14}$ L. J. Richter, C. S. C. Yang, P. T. Wilson, C. A. Hacker, R. D. van Zee, J. J. Stapleton, D. L. Allara, Y. Yao, and J. M. Tour, J. Phys. Chem. B 108, 12547 (2004).

${ }^{15}$ V. Pouthier, C. Ramseyer, and C. Girardet, J. Chem. Phys. 108, 6502 (1998).

${ }^{16}$ B. Busson and A. Tadjeddine, J. Phys. Chem. C 113, 21895 (2009).

${ }^{17}$ W. Gan, D. Wu, Z. Zhang, R.-R. Feng, and H.-F. Wang, J. Chem. Phys. 124, 114705 (2006).

${ }^{18}$ Y. He, Y. Wang, J. Wang, W. Guo, and Z. Wang, Opt. Lett. 41, 874 (2016).

${ }^{19}$ W.-C. Yang and D. K. Hore, J. Chem. Phys. 149, 174703 (2018).

${ }^{20}$ M. Siltanen, S. Cattaneo, E. Vuorimaa, H. Lemmetyinen, T. J. Katz, K. E. S. Phillips, and M. Kauranen, J. Chem. Phys. 121, 1 (2004).

${ }^{21}$ M. Siltanen and M. Kauranen, Opt. Comm. 261, 359 (2006).

${ }^{22}$ R. N. Ward, P. B. Davies, and C. D. Bain, J. Phys. Chem. 97, 7141 (1993).

${ }^{23}$ T. Weidner, J. S. Apte, L. J. Gamble, and D. G. Castner, Langmuir 26, 3433 (2009). 
${ }^{24}$ N. F. Breen, T. Weidner, K. Li, D. G. Castner, and G. P. Drobny, J. Am. Chem. Soc. 131, 14148 (2009).

${ }^{25}$ T. Weidner, N. F. Breen, K. Li, G. P. Drobny, and D. G. Castner, Proc. Natl. Acad. Sci. U.S.A. 107, 13288 (2010).

${ }^{26}$ C. C. Rich, K. A. Lindberg, and A. T. Krummel, J. Phys. Chem. Lett. 8, 1331 (2017).

${ }^{27}$ B. Busson and L. Dalstein, J. Chem. Phys. 149, 034701 (2018).

${ }^{28}$ B. Busson and L. Dalstein, J. Chem. Phys. 149, 154701 (2018).

${ }^{29}$ N. Bloembergen and P. S. Pershan, Phys. Rev. 128, 606 (1962).

${ }^{30}$ N. Bloembergen, R. K. Chang, and C. H. Lee, Phys. Rev. Lett. 16, 986 (1966).

${ }^{31}$ F. Brown, R. E. Parks, and A. M. Sleeper, Phys. Rev. Lett. 14, 1029 (1965).

${ }^{32}$ S. Baldelli, Acc. Chem. Res. 41, 421 (2008).

${ }^{33}$ S. Baldelli, J. Phys. Chem. B 109, 13049 (2005).

${ }^{34}$ S. Baldelli, N. Markovic, P. Ross, Y.-R. Shen, and G. Somorjai, J. Phys. Chem. B 103, 8920 (1999).

${ }^{35}$ Y. Tong, F. Lapointe, M. Thämer, M. Wolf, and R. K. Campen, Angew. Chem. Int. Ed. 56, 4211 (2007).

${ }^{36}$ P. Guyot-Sionnest, Chem. Phys. Lett. 172, 341 (1990).

${ }^{37}$ G. A. Somorjai and K. R. McCrea, Adv. Catalysis 45, 385 (2000).

${ }^{38}$ L. Dalstein, A. Revel, C. Humbert, and B. Busson, J. Chem. Phys. 148, 134701 (2018).

${ }^{39}$ S. A. van den Berg, E. W. M. van der Ham, Q. H. F. Vrehen, and E. R. Eliel, Opt. Lett. 23, 906 (1998).

${ }^{40}$ E. W. M. van der Ham, Q. H. F. Vrehen, E. R. Eliel, V. A. Yakovlev, E. V. Alieva, L. A. Kuzik, J. E. Petrov, V. A. Sychugov, and A. F. G. van der Meer, J. Opt. Soc. Am. B 16, 1146 (1999).

${ }^{41}$ T. F. Heinz, in Nonlinear Surface Electromagnetic Phenomena, edited by H. E. Ponath and G. I. Stegeman (North Holland, Amsterdam, 1991) pp. 353-416.

${ }^{42}$ A. V. Petukhov, Phys. Rev. B 52, 16901 (1995).

${ }^{43}$ J. A. Maytorena, B. S. Mendoza, and W. L. Mochán, Phys. Rev. B 57, 2569 (1998).

${ }^{44}$ V. Mizrahi and J. E. Sipe, J. Opt. Soc. Am. B 5, 660 (1988).

${ }^{45}$ X. Zhuang, P. B. Miranda, D. Kim, and Y. R. Shen, Phys. Rev. B 59, 12632 (1999).

${ }^{46}$ C. Matranga and P. Guyot-Sionnest, J. Chem. Phys. 115, 9503 (2001). 
${ }^{47}$ M. A. Belkin, T. A. Kulakov, K.-H. Ernst, L. Yan, and Y. R. Shen, Phys. Rev. Lett. 85, $4474(2000)$.

${ }^{48}$ E. C. Y. Yan, L. Fu, Z. Wang, and W. Liu, Chem. Rev. 114, 8471 (2014).

${ }^{49}$ M. Kauranen and S. Cattaneo, Prog. Opt. 51, 69 (2008).

${ }^{50}$ J. A. Maytorena, W. L. Mochán, and B. S. Mendoza, Phys. Rev. B 51, 2556 (1995).

${ }^{51}$ S. Yamaguchi, K. Shiratori, A. Morita, and T. Tahara, J. Chem. Phys. 134, 184705 (2011).

${ }^{52}$ S. Sun, C. Tian, and Y. R. Shen, Proc. Nat. Acad. Sci. USA 112, 5883 (2015).

${ }^{53}$ H. Held, A. I. Lvovsky, X. Wei, and Y. R. Shen, Phys. Rev. B 66, 205110 (2002).

${ }^{54}$ D. Maikhuri, S. P. Rurohit, and K. C. Mathur, AIP Adv. 5, 047115 (2015).

${ }^{55}$ X. Wei, S.-C. Hong, A. I. Lvovsky, H. Held, and Y. R. Shen, J. Phys. Chem. B 104, 3349 (2000).

${ }^{56}$ K. Matsuzaki, S. Nihonyanagi, S. Yamaguchi, T. Nagata, and T. Tahara, J. Phys. Chem. Lett. 4, 1654 (2013).

${ }^{57}$ R. Zheng, W. M. Weo, and Q. Shi, Phys. Chem. Chem. Phys. 17, 9068 (2015).

${ }^{58}$ P. Guyot-Sionnest, W. Chen, and Y. R. Shen, Phys. Rev. B 33, 8254 (1986).

${ }^{59}$ F. X. Wang, F. J. Rodríguez, W. M. Albers, R. Ahorinta, J. E. Sipe, and M. Kauranen, Phys. Rev. B 80, 233402 (2009).

${ }^{60}$ J. E. Sipe, V. Mizrahi, and G. I. Stegeman, Phys. Rev. B 35, 9091 (1987).

${ }^{61}$ P. Guyot-Sionnest and Y. R. Shen, Phys. Rev. B 38, 7985 (1988).

${ }^{62}$ B. S. Mendoza, W. L. Mochán, and J. A. Maytorena, Phys. Rev. B 60, 14334 (1999).

${ }^{63}$ K. C. Jena and D. K. Hore, J. Phys. Chem. C 113, 15364 (2009).

${ }^{64}$ K. C. Jena, P. A. Covert, and D. K. Hore, J. Chem. Phys. 134, 044712 (2011).

${ }^{65}$ P. A. Covert, W. A. FitzGerald, and D. K. Hore, J. Chem. Phys. 137, 014201 (2012).

${ }^{66}$ S. Sun, R. Liang, X. Xu, H. Zhu, Y. R. Shen, and C. Tian, J. Chem. Phys. 144, 244711 (2016).

${ }^{67}$ L. Fu, S.-L. Chen, and H.-F. Wang, J. Phys. Chem. B 120, 1579 (2016).

${ }^{68}$ M. Thämer, T. Garling, R. K. Campen, and M. Wolf, J. Chem. Phys. 151, 064707 (2019).

${ }^{69}$ M. A. Hines, J. A. Todd, and P. Guyot-Sionnest, Langmuir 11, 493 (1995).

${ }^{70}$ R. Braun, B. D. Casson, C. D. Bain, E. W. M. van der Ham, Q. H. F. Vrehen, E. R. Eliel, A. M. Briggs, and P. B. Davies, J. Chem. Phys. 110, 4634 (1999). 